\title{
Neuro-Genetic Approach for Detecting Changes in Multitemporal Remotely Sensed Images
}

\author{
Aditi Mandal ${ }^{1}$, Susmita Ghosh ${ }^{2}$, and Ashish Ghosh ${ }^{3}$ \\ 1 Ixia Technologies Pvt. Ltd., Sector V, Kolkata, India \\ maditi04@yahoo.co.in \\ 2 Department of Computer Science and Engineering, Jadavpur University, India \\ susmitaghoshju@gmail.com \\ 3 MIU and CSCR, Indian Statistical Institute, Kolkata, India \\ ash@isical.ac.in
}

\begin{abstract}
In the present work the searching capability of Genetic Algorithms (GAs) is exploited to evolve suitable Hopfield type neural network architectures for optimum change detection of multitemporal remotely sensed images. Experiments carried out on two remote sensing images confirm the effectiveness of the proposed technique.
\end{abstract}

Keywords: Change detection, Hopfield type neural network, Genetic Algorithm, Remote sensing, Multitemporal images.

\section{Introduction}

In remote sensing applications, change detection is the process of identifying differences in the state of an object or phenomenon by analyzing a pair of images acquired on the same geographical area at different times [1]. Two preprocessed images, radiometrically and geometrically [2] corrected, are compared pixel by pixel to generate a third image, called the difference image. Once image comparison is performed, the change detection process can be carried out by adopting either context-insensitive or context-sensitive procedure. Commonly used context-insensitive techniques are based on image or histogram thresholding which do not take into account the spatial correlation between neighboring pixels in the decision process. To overcome this limitation, a distribution free, context-sensitive and unsupervised change detection procedure based on Hopfield network is suggested in [3].

In the aforementioned neural network based change detection procedure [3], each spatial position (pixel) in the difference image is represented by a neuron in the Hopfield network and it is fully connected to its neighboring neurons. But sometimes full connectivity may result in misclassification of a pixel. It is, therefore, necessary to deal with variable connected networks for improving the quality of the change detection map. In this article the searching capability of GAs is exploited to evolve Hopfield type optimum neural network architectures for context-sensitive unsupervised change detection in multitemporal remotely sensed images. 


\section{Hopfield's Model of Neural Networks}

A Hopfield neural network consists of a set of neurons where output of each neuron is fed back to each of the other neurons in the network. There is no self feedback loop, and the synaptic weights are symmetric. Depending on the output values a neuron can take, there are two types of Hopfield models: discrete and continuous.

\subsection{Discrete Model}

In the discrete model, neurons are bipolar i.e., the output $V_{i}$ of neuron $i$ is either +1 or -1 . Hopfield defined the energy function of the network [4] as

$$
E=-\sum_{i=1}^{z} \sum_{j=1, i \neq j}^{z} W_{i j} V_{i} V_{j}-\sum_{i=1}^{z} I_{i} V_{j}+\sum_{i=1}^{z} \theta_{i} V_{i}
$$

where the weight $W_{i j}$ represents the synaptic interconnection strength from neuron $j$ to neuron $i$, and $z$ is the total number of neurons in the network; $\theta_{i}$ is the predefined threshold and $I_{i}$ is the initial input bias applied externally to the neuron $i$. The change of energy $\Delta E$ due to a change of output state of a neuron $i$ by $\Delta V$ is

$$
\Delta E=-\left[\sum_{j=1, i \neq j}^{z} W_{i j} V_{j}+I_{i}-\theta_{i}\right] \Delta V_{i}=-\left[U_{i}-\theta_{i}\right] \Delta V_{i}
$$

Any change in $V_{i}$ makes $\Delta E$ negative. At each iteration, the energy value decreases and the network reaches a stable state when its energy value reaches the minimum [4.

\subsection{Continuous Model}

In this model, the output of a neuron is continuous and $V_{i}$ lies in the range $[-1,+1]$. The energy function $E$ of the continuous model is given by

$$
E=-\sum_{i=1}^{z} \sum_{j=1, i \neq j}^{z} W_{i j} V_{i} V_{j}-\sum_{i=1}^{z} I_{i} V_{j}+\sum_{i=1}^{z} \frac{1}{R_{i}} \int_{0}^{V_{i}} g^{-1}\left(V_{i}\right) d v .
$$

The function $E$ is a Liapunov function, and $R_{i}$ is the total input impedance of the amplifier realizing a neuron $i$. The last term in (3) is the energy loss term, which becomes zero at the high gain region. Therefore, the only stable points of the very high-gain continuous deterministic Hopfield model correspond to the stable points of the discrete stochastic Hopfield model [4]. 


\section{Change Detection Based on Modified Hopfield Neural Network Architecture}

For change detection of two coregistered and radiometrically corrected multispectral images $X_{1}$ and $X_{2}$ of size $p \times q$, acquired over the same area at different times $T_{1}$ and $T_{2}$, at first the difference image $D=l_{m n}, 1 \leq m \leq p, 1 \leq n \leq q$ is obtained by applying the CVA technique [12], where $l_{m n}$ is the gray value of the spatial position $(m, n)$. A neuron is assigned to each $(m, n) \in D$ as introduced in [5]. The spatial correlation between neighboring pixels is modeled by defining the neighborhood systems $N$ of order $d$, for a given position $(m, n)$ as $N^{d}{ }_{m n}=(m, n)+(u, v) ;(u, v) \in N^{d}$. The connection strength between the $(m, n)^{t h}$ and $(u, v)^{t h}$ neurons $W_{m n, u v}=1$ if $(u, v) \in N_{m n}^{d}$; otherwise, $W_{m n, u v}=0$. Each neuron in the network is initialized by external input bias $I_{m n}$ which is computed considering the optimal initialization threshold $t_{0}$ and automatically derived initialization threshold $t_{1}[3]$. The energy function of the network as defined in [5] is as follows

$$
E=-\sum_{m=1}^{p} \sum_{n=1}^{q} \sum_{(u, v) \epsilon N_{m n}^{d}} W_{m n, u v} V_{m n} V_{u v}-\sum_{m=1}^{p} \sum_{n=1}^{q} I_{m n} V_{m n}
$$

In terms of images, the first part of (4) can be seen as the impact of the gray values of the neighboring pixels, whereas the second part can be attributed to the gray value of the pixel under consideration [5]. The minimization of (4) results in a stable state of the network in which changed pixels (with output +1 ) are separated from unchanged ones (with output -1).

\section{Architecture Evolution Using GAs for Change Detection}

The connectivity of a neuron allows the neighborhood information to be used for deciding the class of the corresponding pixel as changed or unchanged. In case, some of the neighbors are not completely corrected (geometrically or radiometrically) in the preprocessing step or the pixel is in boundary region, then full connectivity (which considers maximum neighborhood information) may result in misclassification of the said pixel. It is, therefore, necessary to adopt variable connected networks for improving the performance of change detection. This will also enable us to have less connectivity (i.e., less expensive network) as compared to the fully connected one, besides the improved performance.

Here, each chromosome of a GA [6] represents a network architecture as it is viewed in [7. For a $p \times q$ image, each pixel (neuron) being connected to at most $k$ of its neighbors, the length of the chromosome is $p \times q \times k$. If a neuron is connected to any of its neighbors, the corresponding bit of the chromosome is set to 1, else 0. Each network is allowed to run for change detection as described in the previous section. The energy value obtained at the converged state of a network is taken as the index of fitness of the corresponding chromosome 
(minimum energy corresponds to maximum fitness) for its selection for the next generation. The best chromosome of the final population represents the Hopfield type optimum neural network architecture.

\section{$5 \quad$ Results and Analysis}

To assess the effectiveness of the proposed approach, we consider remote sensing data sets corresponding to geographical area of Mexico which is made up of two multispectral images acquired by the sensor of the Landsat-7 satellite in April 2000 and May 2002 (Figs. 1(a) and 1(b), respectively). Between the two aforementioned acquisition dates, a fire destroyed a large portion of the vegetation in the considered region. The corresponding difference image and the reference map are shown in Figs. 1(c) and 1(d), respectively.

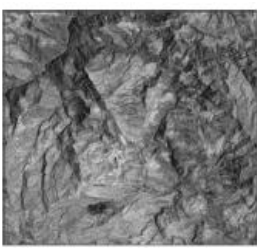

(a)

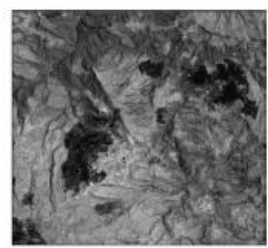

(b)

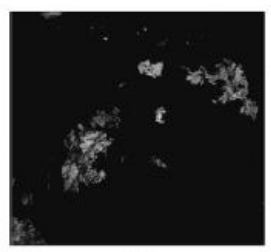

(c)

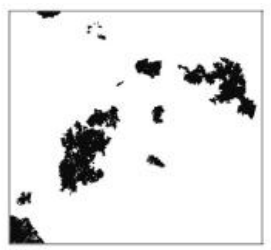

(d)

Fig. 1. Images of Mexico area. (a) Band 4 of the Landsat ETM+ image acquired in April 2000, (b) band 4 of the Landsat ETM+ image acquired in May 2002, (c) corresponding difference image generated by CVA technique, and (d) reference map of changed area.

After analyzing the histogram of the difference image (DI) generated by CVA technique, we observe that the highest peak is in the region of lower gray value. If we consider the properties of the DI, a reasonable assumption can be as follows: a pixel having lower gray value exhibits a high probability of being in unchanged class, whereas a pixel with higher gray value has a high probability of being in changed class. This implies that most of the pixels and their neighbors in the lower gray value region belong to the same area i.e., unchanged area. Hence, for this region maximum neighborhood information can be used for deciding a pixel's class. On the contrary, as nature of the neighbors of rest of the pixels is unknown, use of maximum neighborhood information may lead to their misclassification. So we initialize the population of GA in such a way that if a pixel has lower gray value then the corresponding bits in the chromosome will be 1 with the probability 0.9 ; otherwise, it will be filled up with 0 s and 1 s with equal probability. The population size is kept fixed to 50. The generational replacement technique and elitist model are adopted here along with linear normalization selection 6]. Multipoint crossover is implemented where number of cross-sites is set to 1000 and crossover probability, $p_{c}$, is set to 0.9 . It is expected that in 
the final architecture we have more 1s than 0s because most of the pixels will be connected with more than half of their neighbors to use the contextual information. So we carry out the experiment with two different sets of mutation probabilities $p_{m}$ and $p_{b}$ to preserve $1 \mathrm{~s}$ more than preserving $0 \mathrm{~s}$. We mutate the bit 0 with the probability $p_{m}$ only, whereas bit 1 is mutated with probability $p_{m} \times p_{b}$. For second order connectivity, we have taken $p_{m}=0.01, p_{b}=0.01$. The mutation probability, $p_{m}$, is decreased to 0.001 in case of the first order connectivity as the the length of the chromosome is much less in the first order than in the second order. The network attains stable state around $350^{\text {th }}$ and $200^{\text {th }}$ generations for the first and the second order connectivity, respectively.

In order to establish the effectiveness of the proposed technique, a comparison is carried out between the change detection results produced by the proposed GA based method and those using the Hopfield-Type neural networks (HTNN) [3. Results obtained using both the methods are put in Table 1. It shows that the proposed model always produces less overall error (and a network with less number of connections) as compared to the HTNN counterpart. Also, the second order connectivity is found to be more effective than the first order.

Table 1. Change detection results obtained by the HTNN and the proposed technique

\begin{tabular}{|c|c|c|c|c|c|c|c|c|}
\hline \multirow{2}{*}{$\begin{array}{l}\text { Network } \\
\text { Model used }\end{array}$} & \multirow[t]{2}{*}{ Connectivity } & \multicolumn{2}{|c|}{$\begin{array}{l}\text { Initialization } \\
\text { Threshold }\end{array}$} & \multirow{2}{*}{$\begin{array}{l}\text { Technique } \\
\text { used }\end{array}$} & \multirow{2}{*}{$\begin{array}{l}\text { No. of } \\
\text { Missed } \\
\text { alarms }\end{array}$} & \multirow{2}{*}{$\begin{array}{l}\text { No. of } \\
\text { False } \\
\text { alarms }\end{array}$} & \multirow{2}{*}{$\begin{array}{l}\text { Overall } \\
\text { error }\end{array}$} & \multirow{2}{*}{$\begin{array}{l}\text { Number of } \\
\text { Connections }\end{array}$} \\
\hline & & $\mathrm{t}_{0}$ & $\mathrm{t}_{1}$ & & & & & \\
\hline \multirow{8}{*}{ Discrete } & \multirow{4}{*}{$1^{\text {st }}$} & \multirow{2}{*}{34} & \multirow[b]{2}{*}{ - } & HTNN & 1252 & 1640 & 2892 & 1048576 \\
\hline & & & & Proposed & 1054 & 1749 & 2803 & 1027330 \\
\hline & & \multirow[b]{2}{*}{ - } & \multirow{2}{*}{33} & HTNN & 1102 & 1802 & 2904 & 1048576 \\
\hline & & & & Proposed & 915 & 1878 & 2793 & 1035214 \\
\hline & \multirow{4}{*}{$2^{\text {nd }}$} & \multirow{2}{*}{31} & \multirow[b]{2}{*}{ - } & HTNN & 1010 & 1740 & 2750 & 2097152 \\
\hline & & & & Proposed & 936 & 1711 & 2647 & 2083341 \\
\hline & & \multirow[b]{2}{*}{ - } & \multirow{2}{*}{27} & HTNN & 608 & 2404 & 3012 & 2097152 \\
\hline & & & & Proposed & 489 & 2309 & 2798 & 2085111 \\
\hline \multirow{8}{*}{ Continuous } & \multirow{4}{*}{$1^{\text {st }}$} & \multirow{2}{*}{34} & \multirow[b]{2}{*}{ - } & HTNN & 1035 & 1554 & 2589 & 1048576 \\
\hline & & & & Proposed & 1120 & 1461 & 2581 & 1037125 \\
\hline & & \multirow[b]{2}{*}{ - } & \multirow{2}{*}{31} & HTNN & 660 & 2157 & 2817 & 1048576 \\
\hline & & & & Proposed & 767 & 1907 & 2674 & 1035118 \\
\hline & \multirow{4}{*}{$2^{\text {nd }}$} & \multirow{2}{*}{30} & \multirow{2}{*}{ - } & HTNN & 733 & 2211 & 2944 & 2097152 \\
\hline & & & & Proposed & 766 & 2108 & 2874 & 2077246 \\
\hline & & \multirow[b]{2}{*}{ - } & \multirow{2}{*}{28} & HTNN & 558 & 2707 & 3265 & 2097152 \\
\hline & & & & Proposed & 718 & 2403 & 3121 & 2060882 \\
\hline
\end{tabular}

Figs. 2(a) and 2(b) depict the change detection maps produced by the HTNN and the proposed technique, respectively for the first-order continuous model with $t_{1}=31$. Figs. 2(c) and 2(d), respectively, show the change detection maps obtained using the HTNN and the proposed technique for the second order discrete model with $t_{1}=27$. A visual comparison points out that the proposed approach, due to a proper exploitation of the contextual information, generates a more smooth change detection map compared to the fully connected one. 


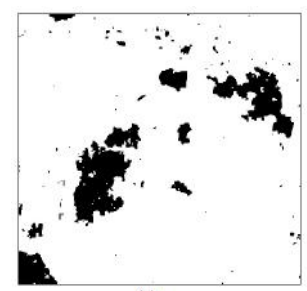

(a)

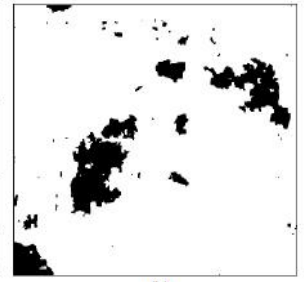

(b)

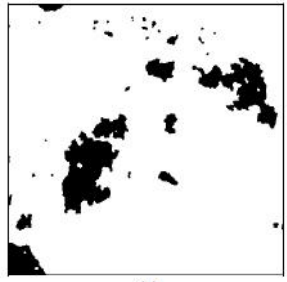

(c)

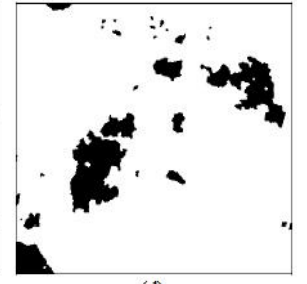

(d)

Fig. 2. Change detection maps obtained using (a) the HTNN technique, (b) the proposed technique for first-order continuous model with $t_{1}=31$ and (c) the HTNN technique, (d) the proposed technique for second-order discrete model with $t_{1}=27$

\section{Conclusion}

Experimental results obtained on multitemporal data sets confirm the effectiveness of the proposed GA based technique. The method always produces less overall change detection error compared to the one achieved using HTNN model. Additionally, the number of connections in the evolved network is always less compared to the corresponding fully connected version.

Acknowledgments. The authors like to thank the Department of Science and Technology (DST), Government of India and University of Trento, Italy, the sponsors of the ITPAR program and Prof. L. Bruzzone, the Italian collaborator of the project, for providing the data.

\section{References}

1. Singh, A.: Digital change detection techniques using remotely sensed data. International Journal of Remote Sensing 10(6), 989-1003 (1989)

2. Richards, J.A., Jia, X.: Remote Sensing Digital Image Analysis. Springer, Berlin (2006)

3. Ghosh, S., Bruzzone, L., Patra, S., Bovolo, F., Ghosh, A.: A context-sensitive technique for unsupervised change detection based on Hopfield type neural networks. IEEE Transactions on Geoscience and Remote Sensing 45(3), 778-789 (2007)

4. Hopfield, J.J.: Neural networks and physical systems with emergent collective computational abilities. Proceedings of the National Academy of Sciences, USA 79, 2554-2558 (1982)

5. Ghosh, A., Pal, N.R., Pal, S.K.: Object background classification using Hopfield type neural network. International Journal of Pattern Recognition and Artificial Intelligence 6(5), 989-1008 (1992)

6. Goldberg, D.E.: Genetic Algorithms in Search, Optimization, and Machine Learning. Pearson Education, London (2003)

7. Pal, S.K., De, S., Ghosh, A.: Designing Hopfield type networks using genetic algorithms and its comparison with simulated annealing. International Journal of Pattern Recognition and Artificial Intelligence 11(3), 447-461 (1997) 